The Astrophysical Journal, 563:L115-L118, 2001 December 20

(C) 2001. The American Astronomical Society. All rights reserved. Printed in U.S.A.

\title{
FIRST CLEAR SIGNATURE OF AN EXTENDED DARK MATTER HALO IN THE DRACO DWARF SPHEROIDAL
}

\author{
Jan T. Kleyna, ${ }^{1}$ Mark I. Wilkinson, ${ }^{1}$ N. Wyn Evans, ${ }^{2}$ and Gerard Gilmore ${ }^{1}$ \\ Received 2001 September 19; accepted 2001 November 9; published 2001 December 7
}

\begin{abstract}
We present the first clear evidence for an extended dark matter halo in the Draco dwarf spheroidal galaxy based on a sample of new radial velocities for 159 giant stars out to large projected radii. Using a two-parameter family of halo models spanning a range of density profiles and velocity anisotropies, we are able to rule out (at about the $2.5 \sigma$ confidence level) halos in which mass follows light. The data strongly favor models in which the dark matter is significantly more extended than the visible dwarf. However, halos with harmonic cores larger than the light distribution are also excluded. When combined with existing measurements of the proper motion of Draco, our data strongly suggest that Draco has not been tidally truncated within $\sim 1 \mathrm{kpc}$. We also show that the rising velocity dispersion at large radii represents a serious problem for modified gravity (MOND).

Subject headings: celestial mechanics — dark matter — galaxies: individual (Draco) —

galaxies: kinematics and dynamics — Local Group — stellar dynamics
\end{abstract}

\section{INTRODUCTION}

The central velocity dispersions of many Local Group dwarf spheroidal (dSph) galaxies are significantly larger than expected for self-gravitating systems (e.g., Mateo 1998). Assuming virial equilibrium, the implied mass-to-light $(M / L)$ ratios reach as high as $\sim 250$, making the dSph galaxies among the most dark matter-dominated systems in the universe. Given the apparent absence of dark matter in globular clusters (e.g., Dirsch \& Richtler 1995), dSph galaxies are also the smallest dark matter-dominated stellar systems in the universe. As such, they have emerged as crucial testing grounds for competing theories of dark matter.

Despite their importance, dynamical models of dSph galaxies to date have been very simple. Most analyses have relied on the use of single-mass isotropic King models, with their associated assumptions that mass follows light and that the stellar velocity distribution is isotropic (although see Pryor \& Kormendy 1991 and Lokas 2001 for more general models). Hitherto, the validity of such assumptions has remained unchallenged because of the small size of the data sets. When only small numbers of radial velocities are available, there is a well-known degeneracy between mass and velocity anisotropy (e.g., Binney $\&$ Tremaine 1987). An increase in the line-of-sight velocity dispersion at large radii may by due to either (1) the presence of large amounts of mass at large radii or (2) tangential anisotropy in the velocity distribution. The primary motivation of this Letter is to break this degeneracy for Draco by means of improved modeling and a larger data set with many more stars in the outer parts.

\section{DATA}

Observations were conducted from 2000 June 23 to 26 at the $4 \mathrm{~m}$ William Herschel Telescope using the AF2/WYFFOS multifiber positioner and spectrograph. A total of 284 stars were observed, spanning the magnitude range of $V \approx 17.0-19.8$. Of these, 159 were Draco members (extending to $25^{\prime}$ ) with spectra of sufficient quality to be included in our dynamical analyses. The median velocity uncertainty for these 159 stars was $1.9 \mathrm{~km} \mathrm{~s}^{-1}$. Of our stars, 62 were previously observed by Ol-

\footnotetext{
${ }^{1}$ Institute of Astronomy, University of Cambridge, Madingley Road, Cambridge, $\mathrm{CB} 3 \mathrm{OHA}$, UK.

${ }^{2}$ Theoretical Physics, University of Oxford, 1 Keble Road, Oxford, OX1 3NP, UK.
}

szewski, Pryor, \& Armandroff (1996); the agreement between our data and the previous data is consistent with a binary fraction of $\sim 40 \%$. Full details of the data are presented in Kleyna et al. (2002).

Previous studies (e.g., Hargreaves et al. 1996) have reported weak evidence of net rotational motion in Draco. $\mathrm{N}$-body simulations show that tidal disruption of $\mathrm{dSph}$ galaxies leads to an apparent rotation of the remnant (Oh, Lin, \& Aarseth 1995; Kroupa 1997; Klessen \& Zhao 2002). In the case of Draco, which is currently at or near pericenter (see $\S 3.3$ ), the expected axis of rotation is perpendicular to the projection of the orbit on the sky. In our data set, we find that Draco appears to rotate at $6 \mathrm{~km} \mathrm{~s}^{-1}$ at a radius of $30^{\prime}$, with a rotation axis position angle (P.A.) of $62^{\circ}$. However, a Monte Carlo analysis shows that this rotation signal is not statistically significant (Kleyna et al. 2002). Furthermore, the solar rest-frame proper motion of Draco (Scholz \& Irwin 1994) implies that the axis for tidally induced apparent rotation should have a P.A. of $138^{\circ} \pm 23^{\circ}$. The $3 \sigma$ disagreement with the observed rotation axis argues against Draco having been significantly influenced by tides, a conclusion supported by the lack of alignment between the P.A. of Draco's major axis $\left(88^{\circ} \pm 3^{\circ}\right.$; Odenkirchen et al. 2001, hereafter OD01) and its orbit.

From our data set it is possible to measure the line-of-sight velocity dispersion profile for a strongly dark matter-dominated $\mathrm{dSph}$ galaxy for the first time. We divide the data into radial bins and use Bayes' theorem to obtain the probability distribution and uncertainties of the velocity dispersion $\left\langle v^{2}\right\rangle^{1 / 2}$ in each radial bin (see Kleyna et al. 2002 for details). The top panel of Figure 1 shows a plot of the radial variation of $\left\langle v^{2}\right\rangle^{1 / 2}$. The velocity dispersion is clearly flat or gently rising with increasing radius. This plot already rules out the best-fit mass-followslight King model (OD01), for which the dispersion falls to zero at $r_{\text {tidal }}=49.4$ and should have fallen to $\sim 0.59$ times the central dispersion (or $\sim 5 \mathrm{~km} \mathrm{~s}^{-1}$ ) by $22^{\prime}$.

\section{MODELING}

\subsection{Jeans Equations}

The Jeans equations for a spherical stellar system lead directly to a model-independent mass estimator (see eqs. [4-55] and [4-56] of Binney \& Tremaine 1987) requiring only knowledge of the velocity anisotropy and the true radial velocity dispersion 

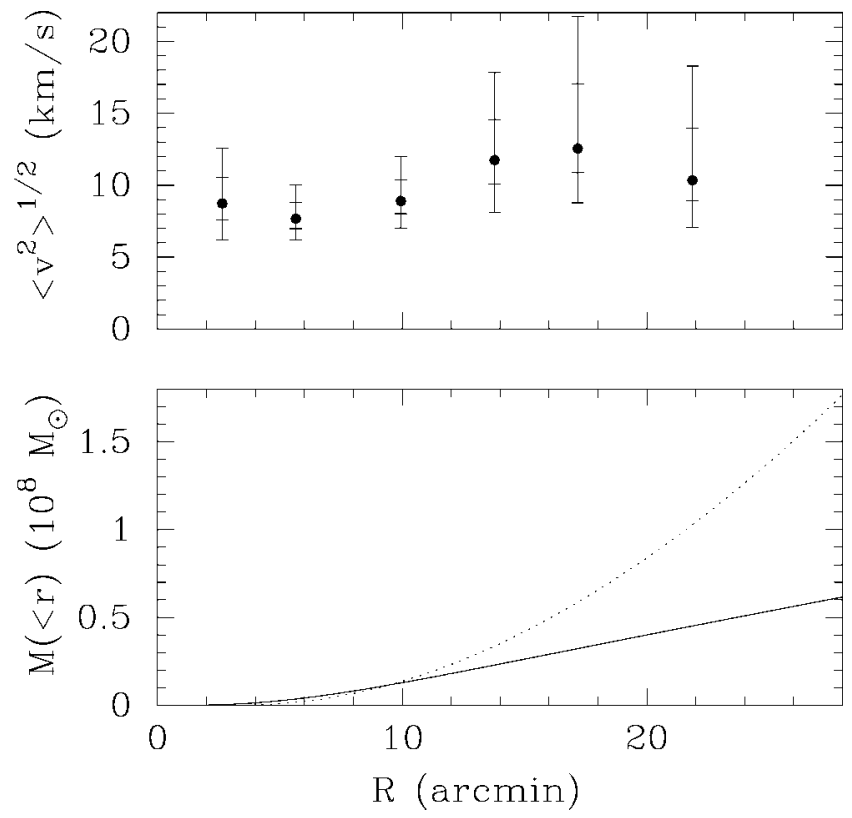

FIG. 1.-Top panel: Line-of-sight velocity dispersion as a function of projected radius $R$. Tick marks show the 1 and $2 \sigma$ error bars assuming a binary fraction of $40 \%$. Bottom panel: Three-dimensional mass profiles for Draco, obtained using the Jeans equations.

$\left\langle v_{r}^{2}\right\rangle$. Assuming spherical symmetry, it is straightforward to obtain $\left\langle v_{r}^{2}\right\rangle$ from the line-of-sight velocity dispersion $\left\langle v^{2}\right\rangle$ using Abel integrals. We consider two dispersion profiles that bracket the true situation: (1) a flat profile with an amplitude of $9.3 \mathrm{~km} \mathrm{~s}^{-1}$ and (2) a profile rising linearly from $8.5 \mathrm{~km} \mathrm{~s}^{-1}$ in the center to $11.25 \mathrm{~km} \mathrm{~s}^{-1}$ at $22^{\prime}$. The light is assumed to follow a Plummer law with a core radius of $r_{0}=9.7$, as this is an excellent fit to the star count data (Kleyna et al. 2002). The solid and dotted curves in the lower panel of Figure 1 show the mass profiles obtained based on these two dispersion profiles, assuming velocity isotropy. The mass enclosed within three core radii ranges from $6.3 \times 10^{7}$ to $18.0 \times 10^{7} M_{\odot}$, implying $M / L$ ratios of $350-1000$, where the $V$-band luminosity of Draco is $1.8 \times$ $10^{5} L_{\odot, V}$ (Irwin \& Hatzidimitriou 1995, hereafter IH95).

\subsection{Halo Models and Distribution Functions}

We now use a two-parameter family of models that spans a range of possibilities for Draco's halo. The halo potential, $\psi(r)$, is given by

$$
\psi(r)= \begin{cases}\psi_{0}\left(1+r^{2} / r_{0}^{2}\right)^{-\alpha / 2} & \text { if } \alpha \neq 0 \\ \left(v_{0}^{2} / 2\right) \log \left(1+r^{2} / r_{0}^{2}\right) & \text { if } \alpha=0 .\end{cases}
$$

The parameter $\alpha$ determines the mass distribution of Draco's halo. If $\alpha=1$, the dark matter has the same density distribution as the light. In models with $\alpha=0$, the halo has an asymptotically flat rotation curve. Finally, when $\alpha=-2$, the visible dwarf lies in the approximately harmonic core of a much larger halo. The distribution functions for dSph galaxy density profiles in potential (1) are derived in Wilkinson et al. (2002). They contain a second parameter $\nu$ that measures the velocity anisotropy. Isotropic models have $\nu=0$, and models with radial (tangential) anisotropy have $\nu>0(\nu<0)$. We generate line profiles from our models by numerically integrating the distribution functions over all transverse velocities. The line profile is then convolved with a binary distribution and Gaussian
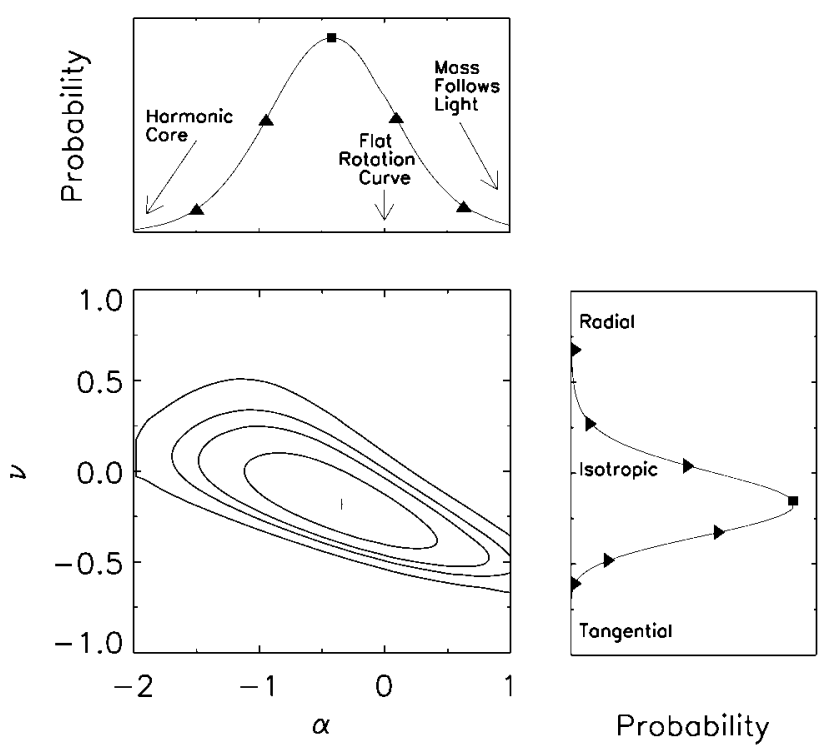

FIG. 2.-Likelihood contours of the fit of our Draco data to the two-parameter $\alpha, \nu$ models of Wilkinson et al. (2002). The contours are at enclosed twodimensional $\chi^{2}$ probabilities of $0.68,0.90,0.95$, and 0.997 . The most likely value is indicated by a plus sign. The top and right panels represent the probability distributions of $\alpha$ and $\nu$, respectively; the median of each distribution is represented by a square, and the triangles show the 1,2 , and $3 \sigma$ limits. The $3 \sigma$ limits are omitted from the projection of $\alpha$ as the upper limit is strongly determined by the cutoff at $\alpha=1$. A $40 \%$ binary fraction is assumed.

velocity measurement uncertainty profile of width $\sigma$ to give $\tilde{L}(v, R, \sigma ; \alpha, \nu)$. The probability of observing an ensemble of points $R_{i}$ with velocity $v_{i}$ and velocity uncertainty $\sigma_{i}$ is then given by the product of the $\tilde{L}\left(v_{i}, R_{i}, \sigma_{i} ; \alpha, \nu\right)$-values.

Figure 2 shows the likelihood contours for the model parameters $\alpha, \nu$ assuming a $40 \%$ binary fraction. The most likely values are $\alpha=-0.34$ and $\nu=-0.18$, indicating a model with significant dark matter at large radii and a tangentially anisotropic velocity distribution. We rule out both a mass-followslight model $(\alpha=1)$ and a harmonic core $(\alpha=-2)$ model at the $\sim 2.5 \sigma$ confidence level. Our best-fit model implies a mass inside three core radii of $8_{-2}^{+3} \times 10^{7} M_{\odot}$. The implied $V$-band $M / L$ ratio of Draco (within three core radii, or $29^{\prime}$ ) is $\sim 440 \pm 240$. This mass profile lies between the two curves in Figure 1 .

All our halo models (eq. [1]) have cores. Cosmological simulations favor cusped halos, such as the Navarro-Frenk-White profile. The present data are insufficient to discriminate strongly between cored and cusped profiles for Draco's dark halounlike the case of the Milky Way galaxy (Binney \& Evans 2001). Cosmological implications of data sets of dSph galaxy radial velocities are discussed elsewhere (Wilkinson et al. 2002, in preparation).

\subsection{Perigalacticon and Tidal Cutoff}

A dSph galaxy in orbit about the Milky Way is truncated by the Galactic tidal field during perigalacticon passages (Oh $\&$ Lin 1992). Based on Sloan Digital Sky Survey images of Draco, OD01 find no evidence for a tidal tail of Draco beyond $50^{\prime}$ and no break in its luminosity profile. Thus, it seems likely that the actual tidal truncation radius of Draco is $\gtrsim 1^{\circ}$.

We use the radial velocity and proper motions to generate possible Draco orbits. The proper motions are given by Scholz $\&$ Irwin (1994) as follows: $\mu_{\alpha} \cos \delta=00^{\prime \prime} 09 \pm 0.05$ century $^{-1}$, $\mu_{\delta}=0.1 \pm 0.05$ century $^{-1}$. The line-of-sight velocity (corrected 
for the solar motion) is $-98 \pm 1 \mathrm{~km} \mathrm{~s}^{-1}$, while the distance of Draco is $82 \pm 7 \mathrm{kpc}$ (Mateo 1998). We represent the Galactic potential by $\psi(r)=v_{0}^{2} \log \left\{\left[\left(a^{2}+r^{2}\right)^{1 / 2}+a\right] / r\right\}$ (e.g., Wilkinson \& Evans 1999), where the scale length is $a \sim$ $170 \mathrm{kpc}$ and the normalization $v_{0}$ is obtained by taking the circular speed to be $220 \mathrm{~km} \mathrm{~s}^{-1}$ at the solar radius. We assume lognormal errors on $a$ with a $3 \sigma$ range from 44 to $489 \mathrm{kpc}$; these limits correspond to masses within $100 \mathrm{kpc}$ of $4.6 \times$ $10^{11} M_{\odot}$ and $1.1 \times 10^{12} M_{\odot}$, respectively. Assuming Gaussian errors on all other observed quantities, we generate sets of initial conditions consisting of a position and velocity for Draco and a scale length for the Milky Way. Each set is integrated in the Galactic potential for a few orbital periods, and the perigalacticon $D_{p}$ and eccentricity $e$ of the orbit are determined. Dynamical friction is unimportant on such short timescales. The contours in Figure 3 show the 1, 2, and $3 \sigma$ limits on the perigalacticon and eccentricity of Draco's orbit. While the distribution of eccentricities is roughly flat in the range [0.3, 0.95], the distribution of perigalacticons is strongly peaked at $\sim 75 \mathrm{kpc}$. This suggests that Draco is currently at or close to perigalacticon.

The tidal radius for a dSph galaxy moving in the above Galactic potential is given by (see King 1962)

$$
r_{t}^{3}=\frac{M_{\mathrm{D}}\left(r_{t}\right) D_{p}^{3}}{M_{\mathrm{G}}(1-e)}\left[\frac{(1+e)^{2}}{2 e v_{0}^{2}}\left(\psi_{p}-\psi_{a}\right)+\frac{a\left(a^{2}+2 D_{p}^{2}\right)}{\left(a^{2}+D_{p}^{2}\right)^{3 / 2}}\right]^{-1},
$$

where $M_{\mathrm{D}}\left(r_{t}\right)$ is the mass of Draco within radius $r_{t}, M_{\mathrm{G}}$ is the total mass of the Galaxy, and $\psi_{p}$ and $\psi_{a}$ are the values of the potential at perigalacticon and apogalacticon. The solid curves in Figure 3 show the variation of $D_{p}$ with $e$ assuming a range of values for $r_{t}$. Only models with $r_{t}>3^{\circ}$ fall within the $1 \sigma$ contour of Draco's perigalacticon and eccentricity derived from its space velocity.

If Draco had recently been subject to strong tidal forces, we would expect significant elongation of the stellar distribution, in conflict with the roundish $(\epsilon=0.3)$ appearance of Draco on the sky and the absence of tidal tails (OD01). Chance alignment effects (e.g., Kroupa 1997) are unlikely to be responsible for Draco's regular appearance. Since any tidal extension of Draco should be aligned with its orbit, elongation approximately along the line of sight would require Draco's orbit to be similarly aligned. However, Monte Carlo sampling of the orbital velocities using the observational error bars rules out alignment within $45^{\circ}$ with $98 \%$ confidence, assuming that the prior probability (with no proper motion information) of the line-of-sight angle is uniform. The absence of statistically significant apparent rotation argues further against line-of-sight elongation.

\section{MODIFIED NEWTON DYNAMICS AND DRACO}

As an alternative to dark matter, Milgrom (1983) proposed a modification to Newton's law of gravity (MOdified Newtonian Dynamics, or MOND) at low accelerations. A number of attempts have been made to apply MOND to the dSph galaxies of the Milky Way (Gerhard 1994; Milgrom 1995). These used only the global velocity dispersion to obtain a MOND $M / L$ ratio and were unable to construct an unambiguous case for or against MOND. Lokas (2001), using published velocities, obtained MOND fits to the dispersion profiles of several dSph galaxies, treating the global MOND acceleration scale as a free parameter.

We model the luminosity density $I(r)$ of Draco using a Plummer sphere with core radius $r_{0}$. The velocity anisotropy param-
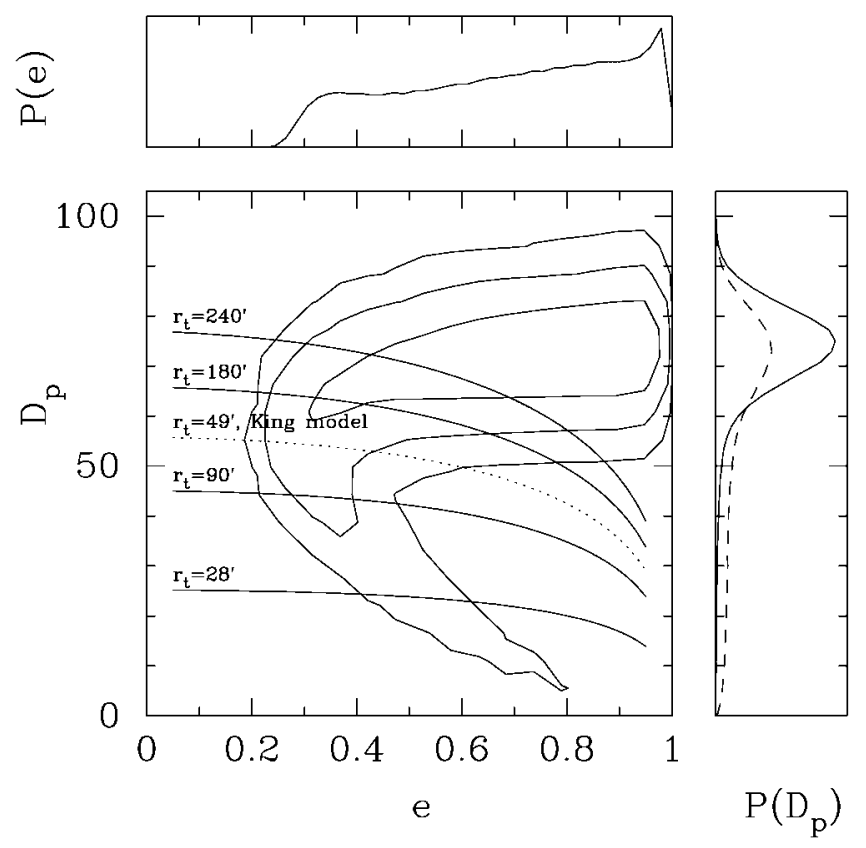

FIG. 3.-Perigalacticon of Draco's orbit vs. orbital eccentricity. Contours are 1, 2, and $3 \sigma$ confidence levels. Overplotted solid curves show the relationship between perigalacticon and eccentricity assuming the labeled values of the tidal radii and our best-fit model for Draco's halo. The dotted curve shows the earlier King model fit to Draco. The solid curves in the top and right panels are the probabilities of $e$ and $D_{p}$, respectively, and the dashed curve in the right panel shows the distribution of $D_{p}$ assuming no proper motion information.

eter $\beta$ (Binney 1981) is assumed to vary with radius as $\beta(r)=(\gamma / 2) r^{2} /\left(r_{0}^{2}+r^{2}\right)$, yielding a velocity distribution that approaches isotropy in the center and becomes increasingly radial (tangential) if $\gamma$ is positive (negative). With these assumptions, the Jeans equations can be integrated to obtain the radial velocity dispersion $\sigma_{r}^{2}(r)$ as

$$
\sigma_{r}^{2}(r)=-\frac{1}{I(r)\left(r_{0}^{2}+r^{2}\right)^{\gamma / 2}} \int_{r}^{\infty}\left(r_{0}^{2}+r^{2}\right)^{\gamma / 2} I(r) g_{\mathrm{M}}(r) d r,
$$

where $g_{\mathrm{M}}(r)$ is the MOND acceleration due to the mass interior to radius $r$. The central projected $V$-band luminosity density $I_{0}$ is $2.2 \times 10^{6} L_{\odot} \mathrm{kpc}^{-2}$ (IH95). Since all of the mass in the MOND picture is provided by the stars, the Newtonian acceleration $g_{\mathrm{N}}(r)$ is given simply by $g_{\mathrm{N}}(r)=-G M_{*}(r) / r^{2}$, where the stellar mass $M_{*}(r)$ is obtained from $I(r)$ and depends on the $M / L$ ratio of the stars. The MOND acceleration is then obtained via $g_{\mathrm{M}}=g_{\mathrm{N}} / \mu\left(g_{\mathrm{M}} / a_{0}\right)$, where $a_{0}=2 \times 10^{-8} \mathrm{~cm} \mathrm{~s}^{-2}$ is the MOND acceleration scale, $\mu(x) \rightarrow 1$ for $x \gg 1$, and $\mu(x) \rightarrow x$ for $x \rightarrow 0$. Our assumed $a_{0}$ is an upper limit to the likely value (Sanders \& Verheijen 1998) and favors models with lower stellar $M / L$ ratios.

Our analysis assumes that Draco is an isolated MOND stellar system. This is justified by noting that for stars between $\sim 1$ '.7 and $\sim 20^{\prime}$, the acceleration due to the baryonic mass of the Milky Way disk $\left(\sim 6 \times 10^{10} M_{\odot}\right)$ at the distance of Draco is smaller than the internal accelerations. Since only $\sim 10 \%$ of our sample lies outside $20^{\prime}$, the assumption of isolation is reasonable. Representing a slightly flattened light distribution by a spherical model has greater impact when applying MOND since all the mass is in the stars. However, in the case of Draco this effect is not significant as our spherical model underestimates 
the mass interior to radius $r$ by $\$ 20 \%$, resulting in $M / L$ ratios that are at most $20 \%$ overestimated.

Using equation (3) and the expression for the velocity anisotropy, we calculate the projected velocity dispersions, from which we obtain the MOND line profiles (assumed Gaussian). These are convolved with the binary velocity distribution and the error distribution to obtain the observable line profiles. Using these profiles, we analyze the velocity data set as in $\S 3.2$, except that the model parameters are now the stellar $M / L$ ratio and the velocity anisotropy. Again we find that some tangential anisotropy is required to reproduce the flat projected velocity dispersion profile. More interestingly, the most likely $M / L$ ratio is 19 , with a $3 \sigma$ lower limit of 8.6. The assumed value of $I_{0}$ implies a total luminosity of $3.2 \times 10^{5} L_{\odot}$ out to $3 r_{0}$, approximately the $2 \sigma$ upper limit of the $V$-band luminosity (IH95). Our lower bound is therefore a true lower limit on the MOND $M / L$ ratio of Draco.

Estimates of the $M / L$ ratio of Draco's stellar population based on comparison with globular clusters have a $3 \sigma$ range of 1-4.3 (Parmentier \& Gilmore 2001; Feltzing, Gilmore, \& Wyse 1999). Even our $3 \sigma$ lower limit on the MOND $M / L$ ratio of Draco is at variance with these values. Thus, even by modifying the law of gravity as prescribed by MOND, we are unable to explain the observed motions of Draco's stars without the presence of significant quantities of dark matter.

\section{DISCUSSION AND CONCLUSIONS}

This Letter has presented a new set of radial velocities for 159 stars in the Draco dSph galaxy. Our data extend to $\sim 25^{\prime}$ and are the first observations to probe the outermost regions of a strongly dark matter-dominated dSph galaxy. The velocity dispersion profile is flat or slowly rising at large radii, which provides the first clear signature of an extended dark matter halo in any dSph galaxy. We analyze these data using both the Jeans equation and halo models with distribution functions. Our best-fit model has a total mass within $3 r_{0} \approx 29^{\prime}$ of $8_{-2}^{+3} \times 10^{7} M_{\odot}$ and a slightly tangentially anisotropic velocity distribution. We are able to rule out the traditional massfollows-light models and extended harmonic core models at about the $2.5 \sigma$ significance level.

From orbit integrations in a Galactic potential, we argue that Draco is currently close to perigalacticon, and its actual tidal radius is greater than $1^{\circ}$, several times the characteristic length scale of the luminous matter. Draco's lack of significant apparent rotation and the significant misalignment of its major axis with its orbit argue against tidal forces having played a major role in its evolution. Our results also have consequences for the modified Newton dynamics (MOND): a Bayesian analysis of the observational data, incorporating radially varying velocity anisotropy, yields a $3 \sigma$ lower limit of 8.6 on the MOND $M / L$ ratio, still requiring the presence of a significant amount of dark matter in Draco.

N. W. E. is supported by the Royal Society, while M. I. W. and J. K. acknowledge help from PPARC. The authors gratefully thank Colin Frayn for help during the observations and Mike Irwin, Hong Sheng Zhao, and the referee Slawomir Piatek for valuable discussions.

\section{REFERENCES}

Binney, J. J. 1981, MNRAS, 196, 455

Binney, J. J., \& Evans, N. W. 2001, MNRAS, 327, L27

Binney, J. J., \& Tremaine, S. 1987, Galactic Dynamics, (Princeton: Princeton Univ. Press)

Dirsch, B., \& Richtler, T. 1995, A\&A, 303, 742

Feltzing, S., Gilmore, G., \& Wyse, R. F. G. 1999, ApJ, 516, L17

Gerhard, O. E. 1994, in Proc. of the ESO/OHP Workshop, Dwarf Galaxies, ed. G. Meylan \& P. Prugniel (Garching: ESP), 335

Hargreaves, J. C., Gilmore, G., Irwin, M. J., \& Carter, D. X. 1996, MNRAS, 282,305

Irwin, M. J., \& Hatzidimitriou, D. 1995, MNRAS, 277, 1354

King, I. 1962, AJ, 67, 471

Kleyna, J. T., Wilkinson, M. I., Evans, N. W., \& Gilmore, G. 2002, MNRAS, submitted (astro-ph/0109450)

Klessen, R. S., \& Zhao, H. 2002 ApJ, 566, 1

Kroupa, P. 1997, NewA, 2, 139
Lokas, E. 2001, preprint (astro-ph/0107479)

Mateo, M. 1998, ARA\&A, 36, 435

Milgrom, M. 1983, ApJ, 270, 365

. 1995, ApJ, 455, 439

Odenkirchen, M., et al. 2001, AJ, 122, 2538(astro-ph/0108100)

Oh, K. S., \& Lin, D. N. C. 1992, ApJ, 386, 519

Oh, K. S., Lin, D. N. C., \& Aarseth, S. J. 1995, ApJ, 442, 142

Olszewski, E. W., Pryor, C., \& Armandroff, T. E. 1996, AJ, 111, 750

Parmentier, G., \& Gilmore, G. 2001, A\&A, 378, 97

Pryor, C., \& Kormendy, J. 1990, AJ, 100, 127

Sanders, R. H., \& Verheijen, M. A. W. 1998, ApJ, 503, 97

Scholz, R.-D., \& Irwin, M. J. 1994, in IAU Symp. 161, Astronomy from WideField Imaging, ed. H. T. MacGillivray et al. (Dordrecht: Kluwer), 535

Wilkinson, M. I., \& Evans, N. W. 1999, MNRAS, 310, 645

Wilkinson, M. I., Kleyna, J. T., Evans, N. W., \& Gilmore, G. 2002, MNRAS, submitted (astro-ph/0109451) 